\title{
CANONICAL MAPS TO TWISTED RINGS
}

\author{
D. ROGALSKI AND J. J. ZHANG
}

\begin{abstract}
If $A$ is a strongly noetherian graded algebra generated in degree one, then there is a canonically constructed graded ring homomorphism from $A$ to a twisted homogeneous coordinate $\operatorname{ring} B(X, \mathcal{L}, \sigma)$, which is surjective in large degree. This result is a key step in the study of projectively simple rings. The proof relies on some results concerning the growth of graded rings which are of independent interest.
\end{abstract}

\section{INTRODUCTION}

Twisted homogeneous coordinate rings (or twisted rings for short) are special noncommutative graded rings, formed from geometric data, which were introduced by Artin and Van den Bergh in $[\mathrm{AV}]$. Given a projective scheme $X$ over a base field $k$, an automorphism $\sigma$ of $X$ and an invertible sheaf $\mathcal{L}$ on $X$, one defines the twisted ring

$$
B(X, \mathcal{L}, \sigma)=\bigoplus_{n=0}^{\infty} \mathrm{H}^{0}\left(X, \mathcal{L} \otimes \sigma^{*} \mathcal{L} \otimes \cdots \otimes\left(\sigma^{n-1}\right)^{*} \mathcal{L}\right)
$$

which has a natural $\mathbb{N}$-graded $k$-algebra structure. If the sheaf $\mathcal{L}$ is $\sigma$-ample (see Section 3 below), then the ring $B(X, \mathcal{L}, \sigma)$ is noetherian and has many other good properties AV] Ke1.

Twisted rings actually first appeared in ATV] (though not named as such) as an important ingredient in the project to classify Artin-Schelter regular algebras of global dimension 3, which leads to a classification of quantum projective planes. Given such a graded regular ring $A$, Artin, Tate and Van den Bergh showed in ATV] that there exists a surjective ring homomorphism $\varphi: A \rightarrow B(X, \mathcal{L}, \sigma)$ for a naturally constructed triple $(X, \mathcal{L}, \sigma)$. This is very useful since the $\operatorname{ring} B(X, \mathcal{L}, \sigma)$, being geometrically defined, may be studied with geometric techniques. Then information may pulled back to the ring $A$. A similar method has been applied with success to certain classes of graded regular rings of global dimension 4 by several authors Va, SV, VV1, VV2].

The goal of this article is to show that the method described above also applies to many other graded rings. We will prove that under quite general conditions on a graded ring $A$, there exists a canonically defined graded ring homomorphism $\varphi: A \rightarrow B(X, \mathcal{L}, \sigma)$, and that this map is always surjective in large degree. As in ATV, the scheme $X$ is constructed as the solution to a certain moduli problem.

2000 Mathematics Subject Classification. 16P90, 16S38, 16W50, 14A22.

Key words and phrases. Graded ring, noncommutative projective geometry, twisted homogeneous coordinate ring, Gelfand-Kirillov dimension, strongly noetherian.

D. Rogalski was partially supported by NSF grant DMS-0202479.

J. J. Zhang was partially supported by NSF grant DMS-0245420 and Leverhulme Research Interchange Grant F/00158/X (UK). 
Let $A$ be a graded algebra generated in degree 1. For each commutative algebra $R$, an $R$-point module for $A$ is a cyclic graded right $A \otimes R$-module $M=\bigoplus_{n=0}^{\infty} M_{n}$ with $M_{0}=R$ and such that $M_{n}$ is a locally free $R$-module of rank 1 for all $n \geq 1$. One may define a functor $F$ from the category of commutative algebras to the category of sets, by sending each commutative algebra $R$ to the set of isomorphism classes of $R$-point modules for $A$. If the functor $F$ is represented by a projective scheme $X$, we call $X$ the point scheme for the ring $A$. By work of Artin and Zhang AZ2 it is known that the point scheme for a graded ring $A$ exists as long as $A$ is strongly noetherian, which means that $A \otimes_{k} R$ is noetherian for all noetherian commutative $k$-algebras $R$. This last condition is satisfied for many important classes of noncommutative graded rings ASZ. Now we may state our main result. Except where otherwise noted, in all of the theorems in this paper the base field $k$ is assumed to be algebraically closed.

Theorem 1.1. Suppose $A$ is a strongly noetherian connected graded $k$-algebra generated in degree 1.

(1) There exists a canonical graded ring homomorphism

$$
\varphi: A \rightarrow B(X, \mathcal{L}, \sigma),
$$

where $X$ is the point scheme for $A$. The kernel of $\varphi$ is equal in large degree to the ideal

$\{x \in A \mid M x=0$ for all $R$-point modules $M$, all commutative algebras $R\}$.

(2) Further, the sheaf $\mathcal{L}$ is $\sigma$-ample, and $\varphi$ is surjective in large degree.

A special case of the above theorem occurs when $A$ is a graded domain of GKdimension 2 which is generated in degree 1 . In this case the canonical map $\varphi$ has already been constructed in AS (although in a different way), and $\varphi$ is known to be an isomorphism in large degree [AS Theorem 0.2]. In fact, such rings $A$ are automatically strongly noetherian ASZ Theorem 4.24], and have graded quotient rings of the form $Q=K\left[t^{ \pm 1} ; \tau\right]$ for some field $K$ of transcendence degree 1 AS, Theorem 0.1]. Thus the following consequence of our main theorem may be viewed as a generalization of $[\mathrm{AS}$, Theorem 0.2] to higher dimensions.

Theorem 1.2. Let $A$ be semiprime, connected graded, generated in degree 1, and strongly noetherian. Suppose that the graded ring of fractions of $A$ has the form $R\left[t^{ \pm 1} ; \tau\right]$, where $R$ is a commutative ring. Then the canonical map $\varphi$ of Theorem 1.1] is an isomorphism in large degree.

We call a locally finite $\mathbb{N}$-graded $k$-algebra $A$ projectively simple if $\operatorname{dim}_{k} A=\infty$ and every nonzero graded ideal $I$ of $A$ satisfies $\operatorname{dim}_{k} A / I<\infty$. Theorem 1.1 also plays a key role in the study of projectively simple rings in the paper RRZ, where we prove the following classification result.

Theorem 1.3. [RRZ, Theorem 0.4 plus Proposition 9.2] Suppose that $A$ is a connected graded domain which is projectively simple, strongly noetherian, is generated in degree 1 , and has at least one $k$-point module.

(1) The canonical map $\varphi: A \rightarrow B(X, \mathcal{L}, \sigma)$ of Theorem 1.1 is an isomorphism in large degree.

(2) $\operatorname{GKdim} A=3$ if and only if $X$ is an abelian surface and $\sigma$ is the translation automorphism $x \mapsto x+b$ for some $b \in X$ such that $\{n b \mid n \in \mathbb{Z}\}$ is Zariskidense in $X$. 
Some other interesting consequences of Theorem 1.1] may be found in Corollary 4.6 below.

Theorem 1.11(1) follows quite formally from the Hilbert scheme theorem [AZ2 and the constructions in ATV. The bulk of the work of this article is directed towards the proof of Theorem 1.12). For this we will develop some results on the growth of graded rings which are of independent interest. See Section 2 below for the relevant definitions of growth and GK-dimension.

Let $R$ be a commutative algebra with algebra automorphism $\sigma$, and let $Q$ be the skew Laurent polynomial ring $R\left[t^{ \pm 1} ; \sigma\right]$. Consider a locally finite $\mathbb{N}$-graded subalgebra $A=\bigoplus_{n=0}^{\infty} V_{n} t^{n} \subset Q$. We call $A$ a big subalgebra of $Q$ if there exists $n \geq 1$ and a unit $u \in V_{n}$ such that $R$ is a localization of its subalgebra $k\left[V_{n} u^{-1}\right]$ at some multiplicative system of nonzerodivisors. The key observation needed for the proof of Theorem 1.12) is that all big subalgebras of $Q$ have closely related growth. For this result the base field $k$ can be arbitrary.

Proposition 1.4. Suppose $A$ is a big subalgebra of $Q$. If $A$ has finite GK-dimension (or subexponential growth), then every locally finite, finitely generated, $\mathbb{N}$-graded subalgebra of $Q$ has finite GK-dimension (respectively subexponential growth).

The proposition is used in the proof of Theorem 1.1 (2) to show that the sheaf $\mathcal{L}$ appearing there is $\sigma$-ample, as follows. We shall see that $B=B(X, \mathcal{L}, \sigma)$ and $\varphi(A)$ are both big subalgebras of the same skew Laurent polynomial ring $Q$, and $\varphi(A)$, being noetherian, has subexponential growth. Then $B$ has subexponential growth, whereas if $\mathcal{L}$ were not $\sigma$-ample then $B$ would have exponential growth.

Although Proposition 1.4 was developed specifically for its application to the proof of Theorem 1.112), it suggests some further interesting questions unrelated to Theorem 1.1

Question 1.5. Suppose that $R$ is a commutative algebra with automorphism $\sigma$ such that there is a locally finite graded big subalgebra $A \subset R\left[t^{ \pm 1} ; \sigma\right]$ with GKdim $A<\infty$.

(1) Does every big locally finite $\mathbb{N}$-graded subalgebra of $R\left[t^{ \pm 1} ; \sigma\right]$ have the same GK-dimension as $A$ ?

(2) Must $R\left[t^{ \pm 1} ; \sigma\right]$ itself have finite GK-dimension as a $k$-algebra?

At the end of the paper we will give a positive answer to this question in a special case. Specifically, we study the case where $R\left[t^{ \pm 1} ; \sigma\right]$ is the graded ring of fractions of some twisted ring $B(X, \mathcal{L}, \sigma)$ where $X$ is integral and $\mathcal{L}$ is $\sigma$-ample.

Theorem 1.6. Let $X$ be an integral projective scheme with automorphism $\sigma$, inducing an automorphism of the function field $K=k(X)$ we also call $\sigma$. Assume that $\mathcal{L}$ is a $\sigma$-ample sheaf on $X$, and put $d=\operatorname{GKdim} B(X, \mathcal{L}, \sigma)<\infty$. Let $Q=K\left[t^{ \pm 1} ; \sigma\right]$.

(1) Every big locally finite $\mathbb{N}$-graded subalgebra $A$ of $Q$ has $\operatorname{GKdim} A=d$.

(2) $d \leq \operatorname{GKdim} Q \leq d+\operatorname{dim} X$.

In general Question [1.5 is still open. As an application of Theorem 1.6 the GK-transcendence degree of $Q$ is computed in Corollary 5.8

\section{GK-TYPE}

Throughout $k$ is a commutative base field, and everything is over $k$. In particular, an algebra or a ring means an algebra over $k$. We refer to $[\mathrm{KL}$ for the topic of growth of algebras. 
For an algebra $A$, we define the Gelfand-Kirillov dimension (or GK-dimension) of $A$ to be

$$
\operatorname{GKdim}(A)=\sup _{V} \varlimsup_{n \rightarrow \infty} \log _{n}\left(\operatorname{dim}_{k} V^{n}\right)
$$

where the supremum is taken over all finite-dimensional $k$-subspaces $V$ of $A$ [GK]. If $A$ is a finitely generated algebra then $\operatorname{GKdim}(A)=\varlimsup \log _{n}\left(\operatorname{dim}_{k} V^{n}\right)$ for any subspace $V$ of $A$ such that $1 \in V$ and $V$ generates $A$ as a $k$-algebra. If $A$ is infinitely generated, then $\operatorname{GKdim}(A)$ is the supremum of $\operatorname{GKdim}(B)$ as $B$ ranges over the finitely generated subalgebras of $A$.

We say that $A$ has exponential growth if

$$
\sup _{V} \varlimsup_{n \rightarrow \infty}\left(\operatorname{dim}_{k} V^{n}\right)^{\frac{1}{n}}>1
$$

where again $V$ ranges over all finite-dimensional $k$-subspaces of $A$. If $A$ is finitely generated and has exponential growth then $\overline{\lim }\left(\operatorname{dim}_{k} V^{n}\right)^{\frac{1}{n}}>1$ holds for any subspace $V$ of $A$ with $1 \in V$ which generates $A$ as an algebra. If $A$ does not have exponential growth then we say it has subexponential growth. Note that if GKdim $A<\infty$, then $A$ has subexponential growth.

The algebras of interest in this paper will be $\mathbb{Z}$-graded algebras $A=\bigoplus_{n=-\infty}^{\infty} A_{n}$. The $\mathbb{Z}$-graded algebra $A$ is $\mathbb{N}$-graded if $A_{n}=0$ for all $n<0$, and locally finite if $\operatorname{dim}_{k} A_{n}<\infty$ for all $n \in \mathbb{Z}$. We call $A$ finitely graded if (i) $A$ is locally finite; (ii) $A$ is $\mathbb{N}$-graded; and (iii) $A$ is finitely generated as an algebra over $k$. If $A_{0}=k, A$ is called connected. If $A$ is locally finite, finitely generated, and $\mathbb{Z}$-graded, then

$$
\operatorname{GKdim} A=\varlimsup_{n \rightarrow \infty} \log _{n}\left(\sum_{i=-n}^{n} \operatorname{dim}_{k} A_{i}\right) .
$$

If both $A_{\geq 0}$ and $A_{\leq 0}$ are locally finite and finitely generated, then GKdim $A=$ $\max \left\{\operatorname{GKdim} A_{\geq 0}, \operatorname{GK} \operatorname{dim} A_{\leq 0}\right\}$. Given a $\mathbb{Z}$-graded algebra $A$, for each $d \geq 1$ we have the $d$ th Veronese ring $A^{(d)}=\bigoplus_{n=-\infty}^{\infty} A_{n d}$, which is again $\mathbb{Z}$-graded.

If the set $S$ of all homogeneous nonzerodivisors of $A$ is an Ore set, then we call the localization $A S^{-1}$ the graded ring of fractions of $A$. If the graded ring of fractions for $A$ exists, and in addition some element $t$ in $A_{1}$ is a nonzerodivisor, then the ring of fractions can be written in the form of a skew Laurent polynomial ring $Q=R\left[t^{ \pm 1} ; \sigma\right]$ for some ring $R$ with automorphism $\sigma$.

Now suppose we are given some skew Laurent polynomial ring $Q=R\left[t^{ \pm 1} ; \sigma\right]$, which we always assume is $\mathbb{Z}$-graded with $R=Q_{0}$ and $t \in Q_{1}$. We want to compare the growth of the various graded subrings of $Q$.

Definition 2.1. Let $R$ be an algebra, and $\sigma: R \rightarrow R$ an algebra automorphism. Let $Q=R\left[t^{ \pm 1} ; \sigma\right]$. Then we say that $Q$ is of finite GK-type if every finitely graded subalgebra $A \subset Q$ has finite GK-dimension. Similarly, we say that $Q$ is of subexponential GK-type if every such $A \subset Q$ has subexponential growth.

We are especially interested in the GK-type of a ring $Q=R\left[t^{ \pm 1} ; \sigma\right]$ where $R$ is commutative. In studying the GK-type we may restrict our attention to those graded subalgebras $A$ of $Q$ which are largest in some sense; intuitively, this means those $A$ for which the graded ring of fractions of $A$ is $Q$ and not some smaller ring. Since graded rings of fractions do not always exist, we will use instead the following notion of largeness for subrings of $Q$, which we already mentioned in the introduction. 
Definition 2.2. Let $Q=R\left[t^{ \pm 1} ; \sigma\right]$ where $R$ is a commutative algebra with algebra automorphism $\sigma$. A locally finite $\left(\mathbb{N}\right.$-) graded subalgebra $A=\bigoplus_{n=0}^{\infty} V_{n} t^{n} \subset Q$ is called a big subalgebra of $Q$ if there is some $n \geq 1$ and an element $u \in V_{n}$ such that (i) $u$ is a unit of $R$; and (ii) $R$ is a localization of its subalgebra $k\left[V_{n} u^{-1}\right]$ at some multiplicative system of nonzerodivisors of $k\left[V_{n} u^{-1}\right]$.

In special cases, for example if $R$ is a field, the concept of a big subalgebra can be formulated in a more intuitive way. The proof of the following alternative characterizations is straightforward.

Lemma 2.3. Let $Q=K\left[t^{ \pm 1} ; \sigma\right]$ with $K$ a field over $k$. Let $A$ be a locally finite graded subalgebra of $Q$.

(1) $A$ is big in $Q$ if and only if the smallest graded simple subring of $Q$ containing $A$ is $K\left[\left(t^{s}\right)^{ \pm 1} ; \sigma^{s}\right]$ for some $s \geq 1$.

(2) If the set of homogeneous nonzerodivisors of $A$ is an Ore set, then $A$ is big in $Q$ if and only if the graded ring of fractions for $A$ is $K\left[\left(t^{s}\right)^{ \pm 1} ; \sigma^{s}\right]$ for some $s \geq 1$.

Our main result concerning GK-type is Proposition [1.4 which shows that if $R$ is commutative, then to prove that $Q=R\left[t^{ \pm 1} ; \sigma\right]$ is of finite (or subexponential) GK-type, it suffices to find a single big subalgebra of $Q$ of finite GK-dimension (respectively subexponential growth). In the proof below, for any subset $W \subset R$ and automorphism $\tau$ of $R$ we use the notation $W^{\tau}=\tau(W)$.

Proof of Proposition 1.4. Let $A=\bigoplus_{n=0}^{\infty} V_{n} t^{n}$ be a big subalgebra of $Q$, and let $B=\bigoplus_{n=0}^{\infty} W_{n} t^{n}$ be an arbitrary finitely graded subring of $Q$. We want to show that if $A$ has finite GK-dimension or subexponential growth, then the same is true of $B$. If $B$ is generated as an algebra in degrees $\leq m$, then the $\operatorname{ring} k+B>_{11}$ is also generated in degrees $\leq m$, and has the same growth as $B$. Replacing $B$ by this algebra we way assume that $B$ is connected. Now $B$ is contained in the algebra generated by $k+W t$ where

$$
W=k+\sum_{n=1}^{m} W_{n} .
$$

Replacing $B$ by this larger algebra we may assume that $B_{n}=\left(B_{1}\right)^{n}$ for $n \geq 1$, so that $B$ is generated in degree 1 .

By the hypothesis that $A$ is big we may find $n \geq 1$ and a unit $u \in V_{n} \subset R$ such that $R$ is a localization of $k\left[V_{n} u^{-1}\right]$. Consider the Veronese subrings $A^{(n)}, B^{(n)}$, which are graded subrings of $Q^{(n)} \cong R\left[\left(t^{n}\right)^{ \pm 1} ; \sigma^{n}\right]$. Since $B$ is finitely generated over $B^{(n)}, B^{(n)}$ and $B$ have the same growth; since $A^{(n)} \subset A$ as ungraded rings, $A^{(n)}$ grows no faster than $A$. Also $A^{(n)}$ is big in $Q^{(n)}$. Thus we may replace $A$ and $B$ by their $n$th Veronese subrings without harm. Then setting $V=V_{1}, W=W_{1}$, and $U=V u^{-1}$, we have $1 \in U$ and $R$ is a localization of $k[U]$.

Thinking of the elements in a basis of $W$ as fractions in $k[U]$ and putting them over a common denominator, we see that we can find an integer $f$ and a unit $u^{\prime} \in R$ such that

$$
W \subset u^{\prime} U^{f}=z V^{f},
$$

where $z=u^{\prime} u^{-f}$ is also a unit. Hence we have

$$
W W^{\sigma} \cdots W^{\sigma^{n}} \subset z \cdots z^{\sigma^{n}}\left(V \cdots V^{\sigma^{n}}\right)^{f}
$$


and thus

$$
\operatorname{dim}_{k} W \cdots W^{\sigma^{n}} \leq \operatorname{dim}_{k}\left(V \cdots V^{\sigma^{n}}\right)^{f} \leq\left(\operatorname{dim}_{k} V \cdots V^{\sigma^{n}}\right)^{f} .
$$

Because $B_{n+1}=\left(W \cdots W^{\sigma^{n}}\right) t^{n+1}$ and $A_{n+1} \supset\left(V \cdots V^{\sigma^{n}}\right) t^{n+1}$, it follows that if $A$ has finite GK-dimension (or subexponential growth), then $B$ also has finite GK-dimension (respectively subexponential growth), as we wished to show.

The following consequence is immediate.

Corollary 2.4. Let $Q=R\left[t^{ \pm 1} ; \sigma\right]$ where $R$ is a commutative algebra. Let $A$ be a finitely graded subalgebra of $Q$. If $A$ has exponential growth, then every finitely graded big subalgebra of $Q$ has exponential growth.

If $R$ is not commutative but only finite over its center, we can understand the GK-type of $Q=R\left[t^{ \pm 1} ; \sigma\right]$ by reducing to the commutative case, using the following result. We include this result for the interested reader, but we will not need to use it later on.

Proposition 2.5. Let $R$ be an algebra with automorphism $\sigma$. Let $S$ be a subalgebra of $R$ such that $S$ is in the center of $R, \sigma$ restricts to an automorphism of $S$, and $R$ is a finite $S$-module. Then $Q=R\left[t^{ \pm 1} ; \sigma\right]$ is of finite (or subexponential) GK-type if and only if $Q^{\prime}=S\left[t^{ \pm 1} ; \sigma\right]$ is of finite (respectively subexponential) GK-type.

Proof. If $Q$ is of finite or subexponential GK-type, then the same obviously holds for the subring $Q^{\prime}$ of $Q$.

For the converse, let $B$ be an arbitrary finitely graded subalgebra of $Q$. We will show that there is a finitely graded subalgebra $A$ of $Q^{\prime}$ with $\operatorname{dim}_{k} B_{n}<d \operatorname{dim}_{k} A_{n}$ for some constant $d>0$ and all $n \geq 0$. This is sufficient to complete the proof.

By the same reductions as in the proof of Proposition 1.4 we may assume that $B$ is connected and generated in degree one, say by $W t$. For the rest of this proof, for any finite-dimensional $k$-subspace $Z$ of $R$ we write $Z_{0}=k$ and $Z_{n}=Z Z^{\sigma} \cdots Z^{\sigma^{n-1}}$ for all $n \geq 1$. With this notation we have $B=\bigoplus_{n \geq 0} W_{n} t^{n}$.

Write $R=\sum_{i=1}^{d} S r_{i}$ for some fixed elements $r_{i} \in R$. Let $T$ be the finitedimensional $k$-span of $\left\{r_{i}\right\}$. We may choose finite-dimensional subspaces $V, U \subset S$ with $1 \in U$ such that $W \subset V T$ and $T T^{\sigma} \subset U T$. Now one proves by induction that

$$
T T^{\sigma} \cdots T^{\sigma^{n-1}} \subseteq T U U^{\sigma} \cdots U^{\sigma^{n-2}} \subseteq T U U^{\sigma} \cdots U^{\sigma^{n-1}}
$$

for all $n \geq 1$. In other words, $T_{n} \subset T U_{n}$ for all $n \geq 1$. Then we have

$$
W_{n} \subseteq T_{n} V_{n} \subseteq T U_{n} V_{n} .
$$

Hence $\operatorname{dim}_{k} W_{n} \leq d \operatorname{dim}_{k} U_{n} V_{n}$ for all $n \geq 0$, where $d=\operatorname{dim}_{k} T$. Define $A \subset Q^{\prime}$ to be the connected graded ring generated in degree 1 by $U V t$. Then $A$ is the desired subring of $Q^{\prime}$ with $\operatorname{dim}_{k} B_{n}<d \operatorname{dim}_{k} A_{n}$ for all $n \geq 0$.

The similarity in the growth of graded subrings of $Q=R\left[t^{ \pm 1} ; \sigma\right]$ which is exhibited by Propositions 1.4 and 2.5 does not necessarily extend to the case where $R$ is not finite over its center, as the following example shows.

Example 2.6. Let $A$ be the graded algebra defined by generators and relations as $A=k\langle x, y, z\rangle /\left(x y-y x-z^{2}, x z-z x, y z-z y\right)$. Then $A$ is one of the standard examples of a 3-dimensional Artin-Schelter regular algebra, and GKdim $A=3$. The graded ring of fractions of $A$ is $Q=D\left[z^{ \pm 1} ; i d_{D}\right]$ where $D$ is the first Weyl 
skew-field. The division ring $D$ contains two elements $f, g \in D$ which generate a free algebra $\mathrm{KL}$, Theorem 8.17]. Thus the subring $A^{\prime}=k[V z] \subset Q$, where $V$ is the $k$-span of $\{1, f, g\}$, has exponential growth, even though $Q$ is the graded ring of fractions of the subring $A$ of finite GK-dimension.

\section{TWisted RINGS OVER NON- $\sigma$-AMPLE $\mathcal{L}$}

Throughout this section let $k$ be an algebraically closed field, $X$ be a projective $k$-scheme, $\sigma$ an automorphism of $X$, and $\mathcal{L}$ an invertible sheaf over $X$. We will show some preliminary results about twisted homogeneous coordinate rings (which we call twisted rings for brevity) for non- $\sigma$-ample invertible sheaves $\mathcal{L}$.

First we recall the definitions from $\mathrm{AV}$. For any sheaf $\mathcal{F}$ on $X$, we use the notation $\mathcal{F}^{\sigma}$ for the pullback $\sigma^{*}(\mathcal{F})$. Set $\mathcal{L}_{0}=\mathcal{O}_{X}$ and $\mathcal{L}_{n}=\mathcal{L} \otimes \mathcal{L}^{\sigma} \otimes \cdots \otimes \mathcal{L}^{\sigma^{n-1}}$ for all $n \geq 1$. The twisted ring associated to the triple $(X, \mathcal{L}, \sigma)$ is

$$
B(X, \mathcal{L}, \sigma)=\bigoplus_{n=0}^{\infty} \mathrm{H}^{0}\left(X, \mathcal{L}_{n}\right),
$$

which has a natural $\mathbb{N}$-graded algebra structure given by the multiplication rule

$$
f \cdot g=f \otimes\left(\sigma^{m}\right)^{*}(g)
$$

for $f \in \mathrm{H}^{0}\left(X, \mathcal{L}_{m}\right), g \in \mathrm{H}^{0}\left(X, \mathcal{L}_{n}\right)$. The invertible sheaf $\mathcal{L}$ is called $\sigma$-ample if for any sheaf $\mathcal{F} \in \operatorname{coh} X, \mathrm{H}^{q}\left(\mathcal{F} \otimes \mathcal{L}_{n}\right)=0$ for all $q>0$ and $n \gg 0$. Other details can be found in $\mathrm{AV}$, Ke1].

Keeler showed that $\sigma$-ampleness depends on a property of the automorphism $\sigma$ called quasi-unipotence Ke1. To define this, let Num $X$ be the quotient of the Picard group of $X$ by the subgroup of divisors numerically equivalent to 0 ; then $\sigma$ induces an action of the finitely generated free abelian group $\operatorname{Num} X$, and $\sigma$ is called quasi-unipotent if the eigenvalues of this action are roots of unity. We will not need the explicit definition of quasi-unipotence below, but only the criterion that Keeler proved: if $\sigma$ is quasi-unipotent, then an invertible sheaf $\mathcal{L}$ is $\sigma$-ample if and only if $\mathcal{L}_{n}$ is ample for some $n$, while if $\sigma$ is not quasi-unipotent then no invertible sheaf on $X$ is $\sigma$-ample Ke1, Theorem 1.3]. When $\mathcal{L}$ is $\sigma$-ample then $B(X, \mathcal{L}, \sigma)$ is a noetherian ring with well-understood properties. Comparatively little is known about twisted rings $B(X, \mathcal{L}, \sigma)$ where $\sigma$ is not quasi-unipotent. The existing knowledge about this case is again due to Keeler, as follows.

Lemma 3.1. Ke1, Proposition 3.7] Suppose $\sigma$ is non-quasi-unipotent. Let $\mathcal{L}$ be an ample invertible sheaf. Then there is an integer $m_{0}$ with the property that for any given $m \geq m_{0}$, one can find constants $C>0$ and $r>1$ such that the graded pieces of $B:=B\left(X, \mathcal{L}^{\otimes m}, \sigma\right)$ satisfy $\operatorname{dim}_{k} B_{n} \geq C r^{n}$. In particular, it follows that for any $m \geq m_{0}$ the ring $B$ is not noetherian.

Remark 3.2. Keeler defines a locally finite $\mathbb{N}$-graded $\operatorname{ring} A=\bigoplus_{n=0}^{\infty} A_{n}$ to have exponential growth if $\varlimsup \lim \left(\sum_{i \leq n} \operatorname{dim}_{k} A_{i}\right)^{1 / n}>1$ [Ke1, Equation (3.3)]. This definition agrees with ours (see Section 2) only if $A$ is finitely generated. An infinitely generated algebra $A$ could well have graded pieces $A_{n}$ growing exponentially in size with $n$, yet have finite GK-dimension under our definitions if it is a directed union of finitely generated subrings of the same finite GK-dimension. Keeler asserts in Ke1, Proposition 3.7] that if $\sigma$ is not quasi-unipotent and $\mathcal{L}$ is ample, then for $m \geq m_{0}$ the ring $B=B\left(X, \mathcal{L}^{\otimes m}, \sigma\right)$ has exponential growth. What is actually 
proven is the exponential growth of graded pieces $\operatorname{dim}_{k} B_{n} \geq C r^{n}$ for some $r>1$ and $C>0$; we take this as the conclusion of Keeler's proposition in Lemma 3.1 above, since it is not enough to imply that $B$ has exponential growth under our definition. To conclude that $B$ is not noetherian for $m \geq m_{0}$, Keeler uses SZ, Theorem 0.1] which states that a locally finite graded ring of exponential growth cannot be noetherian. This conclusion is unaffected by the different definitions of exponential growth, since a noetherian locally finite $\mathbb{N}$-graded algebra would have to be finitely generated as an algebra in any case.

We will generalize Lemma 3.1 to show that if $\sigma$ is not quasi-unipotent but some $\mathcal{L}_{n}$ is very ample, then the $\operatorname{ring} B(X, \mathcal{L}, \sigma)$ already has exponential growth. As we see from the preceding remark, it will be useful first to study finite generation of twisted rings. For this, the theory of Castelnuovo-Mumford regularity will provide a convenient tool. A coherent sheaf $\mathcal{F}$ on $X$ is said to be $m$-regular (with respect to $\mathcal{L})$ if $\mathrm{H}^{i}\left(\mathcal{F} \otimes \mathcal{L}^{\otimes m-i}\right)=0$ for all $i \geq 1$. It is a fact that if a sheaf $\mathcal{F}$ is $m$-regular, then it is also $m^{\prime}$-regular for all $m^{\prime} \geq m$. The regularity of $\mathcal{F}$ is the smallest integer $m$ for which $\mathcal{F}$ is $m$-regular, and is denoted by reg $\mathcal{F}$ once the sheaf $\mathcal{L}$ is fixed.

Now we will see that when $\mathcal{L}$ is "ample enough" the $\operatorname{ring} B(X, \mathcal{L}, \sigma)$ is a finitely generated algebra, regardless of whether $\mathcal{L}$ is $\sigma$-ample or not.

Proposition 3.3. Let $\mathcal{L}$ be an ample invertible sheaf on $X$. Consider the ring $B=B\left(X, \mathcal{L}^{\otimes m}, \sigma\right)$. Then there is some $m_{0} \geq 1$ such that if $m \geq m_{0}$, then the multiplication map $B_{1} \otimes B_{n-1} \rightarrow B_{n}$ is surjective for all $n \geq 1$. In other words, for $m \geq m_{0}$ the ring $B$ is generated in degrees 0 and 1 .

Proof. Fix some arbitrary very ample sheaf $\mathcal{P}$ on $X$ and measure CastelnuovoMumford regularity with respect to $\mathcal{P}$ for the rest of the proof, which we break up into several steps.

Step 1. There exists a constant $C_{1}$ such that for all coherent sheaves $\mathcal{F}, \mathcal{M}$ on $X$ with $\mathcal{M}$ invertible, we have

$$
\operatorname{reg}\left(\mathcal{F} \otimes_{\mathcal{O}_{X}} \mathcal{M}\right) \leq \operatorname{reg} \mathcal{F}+\operatorname{reg} \mathcal{M}+C_{1}
$$

This is proved by Keeler in Ke2 .

Step 2. There is a constant $C_{2}$ such that $\operatorname{reg} \mathcal{N} \leq C_{2}$ for all ample invertible sheaves $\mathcal{N}$ on $X$. This is a consequence of a vanishing Lemma of Fujita Fj, p. 520, Theorem 1].

Step 3. Let $\mathcal{M}$ be an ample invertible sheaf on $X$. Then for any fixed $j \in \mathbb{Z}$ we have $\mathrm{H}^{i}\left(\mathcal{P}^{\otimes j} \otimes \mathcal{M}^{\otimes n}\right)=0$ for $n \gg 0$ and $i>0$ by Serre vanishing. It follows that $\lim _{n \rightarrow \infty} \operatorname{reg} \mathcal{M}^{\otimes n}=-\infty$.

Step 4. For each $m \geq 1$, since $\mathcal{L}^{\otimes m}$ is generated by its global sections we may consider the exact sequence

$$
0 \rightarrow \mathcal{K}_{m} \rightarrow \mathrm{H}^{0}\left(\mathcal{L}^{\otimes m}\right) \otimes \mathcal{O}_{X} \rightarrow \mathcal{L}^{\otimes m} \rightarrow 0 .
$$

Then there is a constant $C_{3}$ such that $\operatorname{reg} \mathcal{K}_{m} \leq C_{3}$ for all $m \geq 1$. To see this, note that for $m \gg 0, \mathcal{L}^{\otimes m}$ is 0 -regular, by step 3. Then for $m \gg 0$ [Ar Lemma 3.1] may be applied to show that $\operatorname{reg} \mathcal{K}_{m} \leq \operatorname{reg} \mathcal{O}_{X}+1$.

Step 5. Consider for all $m \geq 1, n \geq 1$ the sheaf $\mathcal{G}_{m, n}=\mathcal{K}_{m} \otimes\left(\mathcal{L}_{n-1}^{\otimes m}\right)^{\sigma}$, where $\mathcal{K}_{m}$ is defined as in step 4. Since $\mathcal{G}_{m, n}=\mathcal{K}_{m} \otimes\left(\mathcal{L}^{\sigma}\right)^{\otimes m} \otimes \mathcal{N}$ where $\mathcal{N}=\left(\mathcal{L}^{\sigma^{2}} \otimes\right.$ $\left.\cdots \otimes \mathcal{L}^{\sigma^{n}}\right)^{\otimes m}$ is ample, we see from steps 1,2 , and 4 that

$$
\operatorname{reg} \mathcal{G}_{m, n} \leq 2 C_{1}+C_{2}+C_{3}+\operatorname{reg}\left(\mathcal{L}^{\sigma}\right)^{\otimes m} .
$$


Then by step 3 applied with $\mathcal{M}=\mathcal{L}^{\sigma}$, we may choose $m_{0}$ such that $\operatorname{reg} \mathcal{G}_{m, n} \leq 1$ for all $m \geq m_{0}$ and all $n \geq 1$. In particular, we have $\mathrm{H}^{1}\left(\mathcal{G}_{m, n}\right)=0$ for $m \geq m_{0}$ and all $n \geq 1$. Now tensoring the exact sequence of step 4 with $\left(\mathcal{L}_{n-1}^{\otimes m}\right)^{\sigma}$, we get an exact sequence

$$
0 \rightarrow \mathcal{G}_{m, n} \rightarrow \mathrm{H}^{0}\left(\mathcal{L}^{\otimes m}\right) \otimes\left(\mathcal{L}_{n-1}^{\otimes m}\right)^{\sigma} \rightarrow \mathcal{L}_{n}^{\otimes m} \rightarrow 0 .
$$

Taking the long exact sequence in cohomology gives

$$
0 \rightarrow \mathrm{H}^{0}\left(\mathcal{G}_{m, n}\right) \rightarrow \mathrm{H}^{0}\left(\mathcal{L}^{\otimes m}\right) \otimes \mathrm{H}^{0}\left(\left(\mathcal{L}_{n-1}^{\otimes m}\right)^{\sigma}\right) \rightarrow \mathrm{H}^{0}\left(\mathcal{L}_{n}^{\otimes m}\right) \rightarrow \mathrm{H}^{1}\left(\mathcal{G}_{m, n}\right) \rightarrow \ldots
$$

and thus the map $\mathrm{H}^{0}\left(\mathcal{L}^{\otimes m}\right) \otimes \mathrm{H}^{0}\left(\left(\mathcal{L}_{n-1}^{\otimes m}\right)^{\sigma}\right) \rightarrow \mathrm{H}^{0}\left(\mathcal{L}_{n}^{\otimes m}\right)$ is a surjection for $m \geq$ $m_{0}$ and $n \geq 1$. This is exactly the desired claim that the multiplication map $B_{1} \otimes B_{n-1} \rightarrow B_{n}$ is surjective for all $n \geq 1$, for $m \gg 0$.

Next we will prove a needed technical lemma, which is standard in case $X$ is integral, but perhaps not so well-known for general projective schemes $X$. Given any projective scheme $X$, let $\mathcal{K}$ be the sheaf of total quotient rings of $X$ Ha p. 141] and let $R$ be $\mathrm{H}^{0}(\mathcal{K})$, which is called the function ring of $X$ following Nakai. If $X$ is integral, then $R$ is the function field $k(X)$ and $\mathcal{K}$ is simply the constant sheaf on the function field $k(X)$. If $X$ is not integral, $\mathcal{K}$ may not be a constant sheaf. Nakai proved that every invertible sheaf $\mathcal{L}$ on $X$ is equal to a subsheaf of $\mathcal{K}$ Na, Theorem 4]. The automorphism $\sigma$ induces an automorphism of $R$, which we also call $\sigma$. If we fix an embedding $\mathcal{L} \subset \mathcal{K}$ (following $\mathbb{N a}$ Theorem 4]), then for each $i \geq 0$ using the natural isomorphism $\mathcal{K}^{\sigma^{i}} \cong \mathcal{K}$ we get an embedding $\mathcal{L}^{\sigma^{i}} \subset \mathcal{K}$. Also, given any two invertible subsheaves $\mathcal{M}, \mathcal{N} \subset \mathcal{K}$, by thinking of them as Cartier divisors it is clear that $\mathcal{M} \otimes \mathcal{N} \subset \mathcal{K}$ Ha, II.6.13]. Thus we have an embedding $\mathcal{L}_{n} \subset \mathcal{K}$ for each $n \geq 0$ and thus an inclusion $\mathrm{H}^{0}\left(\mathcal{L}_{n}\right) \subset R$. Finally, by adding a placeholder variable $t$, we may think of the twisted $\operatorname{ring} B=B(X, \mathcal{L}, \sigma)$ as the explicit subring $\bigoplus_{n=0}^{\infty} \mathrm{H}^{0}\left(\mathcal{L}_{n}\right) t^{n}$ of $Q=R\left[t^{ \pm 1} ; \sigma\right]$.

Lemma 3.4. Let $\mathcal{L} \subset \mathcal{K}$ be a very ample invertible sheaf, and let $V \subset \mathrm{H}^{0}(\mathcal{L}) \subset R$ be a finite-dimensional $k$-subspace such that the global sections in $V$ generate the sheaf $\mathcal{L}$. Suppose the corresponding map to projective space $\phi: X \rightarrow \mathbb{P}(V)$ is a closed embedding. Then there exists a unit $u \in V$ such that $R$ is the total quotient ring of $k\left[u^{-1} V\right]$.

Proof. Let $d+1=\operatorname{dim}_{k} V$, and write $\mathbb{P}^{d}=\mathbb{P}(V)$. Let $\mathcal{I}$ be the ideal sheaf on $\mathbb{P}^{d}$ defining the closed subscheme $X$. Since $\mathcal{L} \cong \phi^{*}(\mathcal{O}(1))$, we have a homomorphism of graded rings

$$
k\left[z_{0}, \ldots, z_{d}\right]=\bigoplus_{n=0}^{\infty} \mathrm{H}^{0}\left(\mathbb{P}^{d}, \mathcal{O}(n)\right) \stackrel{\theta}{\longrightarrow} \bigoplus_{n=0}^{\infty} \mathrm{H}^{0}\left(X, \mathcal{L}^{\otimes n}\right) t^{n} \subset R\left[t^{ \pm 1}\right] .
$$

The kernel of $\theta$ is $I=\bigoplus_{n=0}^{\infty} \mathrm{H}^{0}\left(\mathbb{P}^{d}, \mathcal{I} \otimes \mathcal{O}(n)\right)$, and the image is the ring $A=$ $\bigoplus_{n=0}^{\infty} V^{n} t^{n}$. Then $A \cong k\left[z_{0}, \ldots, z_{d}\right] / I$, and $A$ has no finite-dimensional ideals Ha, Exercise II.5.10]. Thus $A_{1}$ contains some nonzerodivisor $z=u t$. Let $A_{(z)}$ be the degree 0 piece of the localization of $A$ at $z$. By [Na p. 298], we see that $R$ is the total quotient ring of $A_{(z)}$. Thus $R$ is the total quotient ring of $k\left[V u^{-1}\right] \subset R$, where $u \in V$ is a unit in $R$, as required.

We are now ready to prove our generalization of Lemma 3.1

Proposition 3.5. Let $\sigma$ be a non-quasi-unipotent automorphism of $X$. 
(1) Every big locally finite $\mathbb{N}$-graded subalgebra of $R\left[t^{ \pm 1} ; \sigma\right]$ has exponential growth.

(2) Let $\mathcal{L}$ be an invertible sheaf on $X$ such that $\mathcal{L}_{n}$ is very ample for some $n \geq 1$. Then $B(X, \mathcal{L}, \sigma)$ has exponential growth and is not noetherian.

Proof. (1) Let $\mathcal{L}$ be a very ample invertible sheaf on $X$ with an embedding $\mathcal{L} \subset \mathcal{K}$. By Lemma 3.1] for $m \gg 0$ the $\operatorname{ring} B=B\left(X, \mathcal{L}^{\otimes m}, \sigma\right)$ has the property that $\overline{\lim }\left(\operatorname{dim}_{k} B_{n}\right)^{1 / n}>1$. By Proposition 3.3 above, for $m \gg 0$ we also know that $B$ is finitely generated; together these facts imply that $B$ has exponential growth for $m \gg 0$. Setting $\mathcal{L}^{\prime}=\mathcal{L}^{\otimes m} \subset \mathcal{K}$, we may write $B=\bigoplus_{n=0}^{\infty} \mathrm{H}^{0}\left(X, \mathcal{L}_{n}^{\prime}\right) t^{n} \subset$ $Q:=R\left[t^{ \pm 1} ; \sigma\right]$. By Corollary 2.4 every big finitely graded subalgebra of $Q$ has exponential growth. Since every big locally finite $\mathbb{N}$-graded subalgebra of $Q$ contains a finitely graded big subalgebra, the result follows.

(2) Now let $\mathcal{L}$ be an invertible sheaf on $X$ such that $\mathcal{L}_{n}$ is very ample for some $n$. Fix an embedding $\mathcal{L} \subset \mathcal{K}$ and let $B=B(X, \mathcal{L}, \sigma) \cong \bigoplus_{n=0}^{\infty} \mathrm{H}^{0}\left(\mathcal{L}_{n}\right) t^{n} \subset Q$. Setting $V_{n}=\mathrm{H}^{0}\left(\mathcal{L}_{n}\right) \subset R$, since $\mathcal{L}_{n}$ is very ample the sections $V_{n}$ generate $\mathcal{L}_{n}$, and also the induced map to projective space $X \rightarrow \mathbb{P}\left(V_{n}\right)$ is a closed embedding. Then it follows by Lemma 3.4 that there is a unit $u \in V_{n}$ such that $R$ is the total quotient ring of $k\left[u^{-1} V_{n}\right]$. So by definition $B$ is a big subalgebra of $Q$. Then $B$ has exponential growth by part (1). By [SZ, Theorem 0.1], $B$ is not noetherian.

We note that Proposition 3.5(2) gives a partial answer to [Ke1, Question 3.8], which asks if given a non-quasi-unipotent automorphism $\sigma$ and an ample invertible sheaf $\mathcal{L}$, whether $B(X, \mathcal{L}, \sigma)$ must have exponential growth. We do not know in the non-quasi-unipotent case if given an ample $\mathcal{L}$ there is always some $n \geq 1$ such that $\mathcal{L}_{n}$ is very ample.

\section{The Canonical map $\varphi$}

In this section we assume that $k$ is algebraically closed since we will quote some results from [ATV] and [AZ2] which are under this assumption. A module means a right module. We begin this section with a review of the results of [ATV, §3]. We refer the reader to that paper for more complete details.

Let $A$ be a graded algebra generated in degree 1 and let $\operatorname{dim}_{k} A_{1}=d+1$. Let $T=k\left\langle x_{0}, x_{1}, \ldots, x_{d}\right\rangle$ be the free algebra, and fix a presentation $A \cong T / I$. Let $V=\left(T_{1}\right)^{*}=\operatorname{Hom}_{k}\left(T_{1}, k\right)$. Any $f \in T_{n}$ determines an element in $\left(V^{\otimes n}\right)^{*}$, and thus an element $\widetilde{f} \in \mathrm{H}^{0}\left(\mathbb{P}^{\times n}, \mathcal{O}(1,1, \ldots, 1)\right)$, where $\mathbb{P}^{\times n}$ is an abbreviation for $(\mathbb{P}(V))^{\times n}$. Then $\tilde{f}$ determines a vanishing locus in $\mathbb{P}^{\times n}$. Now for each $n \geq 0$, we let $X_{n} \subset \mathbb{P}^{\times n}$ be the common locus of vanishing of all $\left\{\tilde{f} \mid f \in I_{n}\right\}$. Also for each $n \geq 0$ let $i_{n}: X_{n} \rightarrow \mathbb{P}^{\times n}$ be the inclusion map, and set $\mathcal{M}_{n}=i_{n}^{*} \mathcal{O}(1,1, \ldots 1)$. Now for any $1 \leq m<n$ we define a morphism of schemes $\phi_{n, m}: X_{n} \rightarrow X_{m}$ by restricting the morphism $\pi_{1}: \mathbb{P}^{\times n} \rightarrow \mathbb{P}^{\times m}$ which is the projection onto the first $m$ coordinates. We also define a morphism of schemes $\psi_{n, m}: X_{n} \rightarrow X_{m}$ by restricting the morphism $\pi_{2}: \mathbb{P}^{\times n} \rightarrow \mathbb{P}^{\times m}$ which is the projection onto the last $m$ coordinates. We use the abbreviations $\phi_{n+1, n}=\phi_{n}$ and $\psi_{n+1, n}=\psi_{n}$.

The schemes just constructed are closely related to the point modules for the ring $A$. For any commutative algebra $R$ and $n \geq 0$, a truncated $R$-point module of length $n+1$ is a graded cyclic right $A \otimes R$-module $M=\bigoplus_{i=0}^{n} M_{i}$ where $M_{0}=R$ and each $M_{i}$ for $1 \leq i \leq n$ is a locally free $R$-module of rank 1 . As we defined in the introduction, an $R$-point module is defined similarly, by letting $n=\infty$. A 
$k$-point module is simply called a point module. Then by ATV Proposition 3.9], the scheme $X_{n}$ represents the functor from commutative $k$-algebras to sets which sends an algebra $R$ to the set of isomorphism classes of truncated $R$-point modules of length $n+1$. Moreover, in case the maps $\phi_{n}: X_{n+1} \rightarrow X_{n}$ are isomorphisms for all $n \geq n_{0}$, then $X=X_{n_{0}}$ is the point scheme for $A$ as defined in the introduction; that is, $X$ represents the functor sending an algebra $R$ to the set of isomorphism classes of $R$-point modules.

In the next lemma we gather together some further formal consequences of the definitions above, which are also largely a restatement of results in ATV].

Lemma 4.1. Keep the notations introduced above.

(1) There is a graded ring homomorphism

$$
\tilde{\theta}: T \cong \bigoplus_{n=0}^{\infty} \mathrm{H}^{0}\left(\mathbb{P}^{\times n}, \mathcal{O}(1,1, \ldots, 1)\right) \rightarrow B^{\prime}=\bigoplus_{n=0}^{\infty} \mathrm{H}^{0}\left(X_{n}, \mathcal{M}_{n}\right)
$$

defined by pullback of sections, and $\widetilde{\theta}$ factors through $I$ to give a map $\theta$ : $A \rightarrow B^{\prime}$. An element $x \in T_{n}=\mathrm{H}^{0}\left(\mathbb{P}^{\times n}, \mathcal{O}(1,1, \ldots, 1)\right)$ is in $(\operatorname{ker} \widetilde{\theta})_{n}$ if and only if $i_{n}^{*}(x)=0$.

(2) Let $J=\operatorname{ker} \theta \subset A$. Then for each $n \geq 0, a \in J_{n}$ if and only if $\left(M_{0}\right) a=0$ for all truncated $R$-point modules $M$ of length $n+1$ over $A$, for all commutative $k$-algebras $R$.

Proof. (1) The ring structure on $B^{\prime}$ is described in ATV p. 48], but we will also describe it briefly here. Given $m, n \geq 1$ we have an isomorphism of sheaves on $X_{n+m}$

$$
\phi_{m+n, m}^{*}\left(\mathcal{M}_{m}\right) \otimes \psi_{m+n, n}^{*}\left(\mathcal{M}_{n}\right) \cong \mathcal{M}_{m+n},
$$

and thus a map

$$
\mathrm{H}^{0}\left(X_{m}, \mathcal{M}_{m}\right) \otimes \mathrm{H}^{0}\left(X_{n}, \mathcal{M}_{n}\right) \stackrel{\phi^{*} \otimes \psi^{*}}{\longrightarrow} \mathrm{H}^{0}\left(X_{m+n}, \mathcal{M}_{m+n}\right) .
$$

This gives the multiplication maps $B_{m}^{\prime} \otimes B_{n}^{\prime} \rightarrow B_{m+n}^{\prime}$ for each $m, n$.

The map $\widetilde{\theta}$ is defined in degree $n$ by pulling back sections via the embedding $i_{n}: X_{n} \rightarrow \mathbb{P}^{\times n}$. That this $\widetilde{\theta}$ defines a ring homomorphism factoring through $I$ is ATV Proposition 3.20]. It is immediate from the definition of $\widetilde{\theta}$ as a pullback that $x \in(\operatorname{ker} \widetilde{\theta})_{n}$ if and only if $i_{n}^{*}(x)=0$.

(2) By [ATV Proposition 3.9], for every commutative algebra $R$ there is a bijection between $\operatorname{Hom}_{k-\text { schemes }}\left(\operatorname{Spec} R, X_{n}\right)$ and the set of isomorphism classes of truncated $R$-point modules of length $n+1$ for $A$. One way to make the correspondence explicit is to use the universal truncated point module of length $n+1$ (see [ATV p. 47]). This is a coherent sheaf on $X_{n}$ given by

$$
\mathcal{N}=\bigoplus_{j=0}^{n} \mathcal{N}_{j} \quad \text { where } \quad \mathcal{N}_{j}=i_{n}^{*} \mathcal{O}(1,1, \ldots, 1,0,0, \ldots, 0) .
$$

Note that $\mathcal{N}_{0}=\mathcal{O}_{X_{n}}$. Fix some $j$ with $1 \leq j \leq n$. We have maps

$$
A_{j} \stackrel{\theta_{j}}{\rightarrow} \mathrm{H}^{0}\left(X_{j}, \mathcal{M}_{j}\right) \stackrel{\phi_{n}^{*}, j}{\rightarrow} \mathrm{H}^{0}\left(X_{n}, \mathcal{N}_{j}\right)
$$

and thus we get a morphism of sheaves on $X_{n}$

$$
\mathcal{N}_{0} \otimes_{k} A_{j} \rightarrow \mathcal{O}_{X_{n}} \otimes_{k} \mathrm{H}^{0}\left(X_{n}, \mathcal{N}_{j}\right) \rightarrow \mathcal{N}_{j} .
$$


Now given a commutative algebra $R$ and a $k$-morphism $\rho: \operatorname{Spec} R \rightarrow X_{n}$, we can pull back via $\rho$, and we get a morphism of sheaves on $\operatorname{Spec} R$

$$
\rho^{*}\left(\mathcal{N}_{0}\right) \otimes_{k} A_{j} \rightarrow \rho^{*}\left(\mathcal{N}_{j}\right) .
$$

Then the truncated $R$-point module of length $n+1$ corresponding to the map $\rho$ is simply $M=\rho^{*} \mathcal{N}$; note that $M_{0}=R$ and that the maps (4.2) determine the $A$-module structure on $M$.

There is a canonical generator $1 \in R=M_{0}$ for the truncated point module $M$. Then given $a \in A_{n}$, if we lift $a$ to some $\widetilde{a} \in T_{n}$, then we see that $\left(M_{0}\right) a=0$ if and only if $\rho^{*} i_{n}^{*}(\widetilde{a})=0$.

Now we see from part (1) that $a \in(\operatorname{ker} \theta)_{n}$ if and only if given any lift $\widetilde{a} \in T_{n}$ of $a$, we have $i_{n}^{*}(\widetilde{a})=0$. But the section $i_{n}^{*}(\widetilde{a}) \in \mathrm{H}^{0}\left(X_{n}, \mathcal{M}_{n}\right)$ is zero if and only if $\rho^{*} i_{n}^{*}(\widetilde{a})=0$ for all commutative algebras $R$ and all maps $\rho: \operatorname{Spec} R \rightarrow X_{n}$, if and only if $\left(M_{0}\right) a=0$ for all truncated $R$-point modules $M$ of length $n+1$ by the explicit correspondence given above.

We need one last ingredient for the proof of the main theorem. The following result of Artin and Stafford shows that under certain conditions, a subring $A$ of a twisted $\operatorname{ring} B$ must be equal to $B$ in large degree.

Lemma 4.3. AS Theorem 4.1] Suppose that $B=B(X, \mathcal{L}, \sigma)$ where $\mathcal{L}$ is $\sigma$-ample. Let $A$ be a graded subring of $B$, and suppose that for $n \gg 0$ the sections in $A_{n} \subseteq$ $\mathrm{H}^{0}\left(\mathcal{L}_{n}\right)$ generate the sheaf $\mathcal{L}_{n}$, and that the map to projective space $X \rightarrow \mathbb{P}\left(A_{n}\right)$ determined by a $k$-basis for $A_{n}$ is a closed embedding. Then $A_{n}=B_{n}$ for $n \gg 0$.

Proof. This is a slight restatement of [AS Theorem 4.1], which requires in addition to our hypotheses that $\mathcal{L}$ is also $\sigma^{-1}$-ample, and that $A$ has subexponential growth. But since $\mathcal{L}$ is $\sigma$-ample, $\mathcal{L}$ must also be $\sigma^{-1}$-ample Ke1, Corollary 5.1], and $B$ has finite GK-dimension [Ke1 Theorem 6.1], so $A$ does as well.

We can now prove our most general result about when maps to twisted rings exist and are surjective in large degree.

Theorem 4.4. Let $A$ be a connected graded algebra of subexponential growth which is generated in degree 1 , and let $X_{n}, i_{n}, \phi_{n}, \psi_{n}$, and $\mathcal{M}_{n}$ be defined for each $n \geq 0$ as at the beginning of this section. Assume that there exists some $n_{0}$ such that for all $n \geq n_{0}$, both $\phi_{n}$ and $\psi_{n}$ are isomorphisms of schemes. Write $X=X_{n_{0}}$, $\sigma=\psi_{n_{0}}\left(\phi_{n_{0}}\right)^{-1}$, and let $\mathcal{L}=i_{n_{0}}^{*} \mathcal{O}(1,0,0, \ldots, 0)$. Then

(1) There is a ring homomorphism $\varphi: A \rightarrow B=B(X, \mathcal{L}, \sigma)$.

(2) Let $J$ be the ideal of $A$ defined as

$J=\{x \in A \mid M x=0$ for all $R$-point modules $M$, all commutative algebras $R\}$.

Then $J$ is equal to $\operatorname{ker} \varphi$ in all degrees $\geq n_{0}$.

(3) The sheaf $\mathcal{L}$ is $\sigma$-ample on $X$, and $\varphi$ is surjective in large degree.

(4) The homomorphism in part (1) is canonically determined by the ring $A$.

Proof. (1) We have the homomorphism of graded rings

$$
\theta: A \cong T / I \rightarrow B^{\prime}=\bigoplus_{n=0}^{\infty} \mathrm{H}^{0}\left(X_{n}, \mathcal{M}_{n}\right)
$$


given by Lemma 4.11). With our assumption that the maps $\phi_{n}$ and $\psi_{n}$ are isomorphisms for $n \geq n_{0}$, we will show there is also a ring homomorphism

$$
\rho: B^{\prime}=\bigoplus_{n=0}^{\infty} \mathrm{H}^{0}\left(X_{n}, \mathcal{M}_{n}\right) \rightarrow B=\bigoplus_{n=0}^{\infty} \mathrm{H}^{0}\left(X, \mathcal{L}_{n}\right)=B(X, \mathcal{L}, \sigma)
$$

where $\mathcal{L}_{n}=\mathcal{L} \otimes \mathcal{L}^{\sigma} \otimes \cdots \otimes \mathcal{L}^{\sigma^{n-1}}$ on $X$. Let $n \geq 0$ be arbitrary. If $n>n_{0}$ then we set $\tau=\left(\phi_{n, n_{0}}\right)^{-1}$, which makes sense since $\phi_{n, n_{0}}=\phi_{n_{0}} \phi_{n_{0}+1} \ldots \phi_{n}$ is an isomorphism; otherwise $n \leq n_{0}$ and we set $\tau=\phi_{n_{0}, n}$. In any case $\tau$ is a morphism from $X_{n_{0}}$ to $X_{n}$.

Now we claim that for each $0 \leq m \leq n-1$ we have

$$
\mathcal{L}^{\sigma^{m}}=\tau^{*} i_{n}^{*} \mathcal{O}(0, \cdots, 0, \stackrel{m+1}{1}, 0, \cdots, 0) .
$$

Let us outline the proof the claim for the case $n=n_{0}$, so that $\tau$ is the identity; The proof for other $n$ is very similar and is left for the reader. For convenience, we write $\psi=\psi_{n_{0}}, \phi=\phi_{n_{0}}, i=i_{n_{0}}$, and $j=i_{n_{0}+1}$. For $m=0$, 4.5.5 is just the definition of $\mathcal{L}$.

By definition, we have $\sigma=\psi \phi^{-1}$. Also, $\phi, \psi: X_{n_{0}+1} \rightarrow X_{n_{0}}$ are defined as the restrictions of the two maps $\pi_{1}, \pi_{2}: \mathbb{P}^{\times n_{0}+1} \rightarrow \mathbb{P}^{\times n_{0}}$ which project onto the first (respectively, last) $n_{0}$ coordinates. This implies that $\pi_{1} j=i \phi$ and $\pi_{2} j=i \psi$. Thus altogether we have

$$
\begin{aligned}
\mathcal{L}^{\sigma}=\sigma^{*} \mathcal{L} & =\left(\phi^{-1}\right)^{*} \psi^{*} i^{*} \mathcal{O}(1,0, \cdots, 0) \\
& =\left(\phi^{-1}\right)^{*} j^{*} \pi_{2}^{*} \mathcal{O}(1,0, \cdots, 0) \\
& =\left(\phi^{-1}\right)^{*} j^{*} \pi_{1}^{*} \mathcal{O}(0,1,0, \cdots, 0) \\
& =\left(\phi^{-1}\right)^{*} \phi^{*} i^{*} \mathcal{O}(0,1,0, \cdots, 0) \\
& =i^{*} \mathcal{O}(0,1,0, \cdots, 0)
\end{aligned}
$$

which verifies 4.5) for $m=1$. Now 4.5 may be proven for arbitrary $m$ with $0 \leq m \leq n-1$ by induction. The proof of the induction step is analogous to the preceding calculation and is omitted.

Given (4.5), since tensor product of sheaves commutes with pullback, we see that

$$
\mathcal{L}_{n}=\mathcal{L} \otimes \mathcal{L}^{\sigma} \otimes \cdots \otimes \mathcal{L}^{\sigma^{n-1}}=\tau^{*} i_{n}^{*}(\mathcal{O}(1,1, \cdots, 1))=\tau^{*} \mathcal{M}_{n}
$$

and thus there is a map

$$
\mathrm{H}^{0}\left(X_{n}, \mathcal{M}_{n}\right) \stackrel{\tau^{*}}{\rightarrow} \mathrm{H}^{0}\left(X_{n_{0}}, \tau^{*} \mathcal{M}_{n}\right) \cong \mathrm{H}^{0}\left(X, \mathcal{L}_{n}\right) .
$$

This defines the map $\rho$ in degree $n$. Note that if $n \geq n_{0}$, then $\tau$ is an isomorphism and so $\rho$ is an isomorphism in degrees $\geq n_{0}$. To check that $\rho$ is a ring homomorphism is a formality left to the reader. Finally, we get the desired morphism $\varphi$ by letting $\varphi=\rho \circ \theta$.

(2) Let $\varphi=\rho \circ \theta$ be as constructed in part (1). It is easy to see that the $J$ given in the statement of part (2) is an ideal. If $a \in(\operatorname{ker} \theta)_{n}$, then by Lemma 4.1(2), $\left(M_{0}\right) a=0$ for all truncated $R$-point modules $M$ of length $n+1$ and all commutative algebras $R$. If $N=\bigoplus_{i=0}^{\infty} N_{i}$ is any $R$-point module, then given any $m \geq 0$ the graded piece $N_{m}$ is a locally free $R$-module, so there is a locally free $R$-module $S$ with $S \otimes_{R} N_{m} \cong R$. Then $M=\bigoplus_{i=0}^{n} S \otimes_{R} N_{i+m}$ is a truncated $R$-point module of length $n+1$, and so $M_{0} a=0$. This implies that $N_{m} a=0$. Since $m$ was arbitrary, 
we have $N a=0$ and $a \in J$. Because the map $\rho$ is an isomorphism in degrees $\geq n_{0}$, we see that for $n \geq n_{0}$, if $a \in(\operatorname{ker} \varphi)_{n}$ then $a \in(\operatorname{ker} \theta)_{n}$ and thus $a \in J$.

Conversely, suppose that $a \in J_{n}$ with $n \geq n_{0}$. Given any truncated $R$-point module $M$ of length $n+1$, since $\phi_{n}$ is an isomorphism for $n \geq n_{0}$ there exists an $R$-point module $N=\bigoplus_{i=0}^{\infty} N_{i}$ with $M \cong \bigoplus_{i=0}^{n} N_{i}$. Since $N a=0,\left(M_{0}\right) a=0$. Thus $a \in \operatorname{ker} \theta$ by Lemma 4.11(2) and so $a \in \operatorname{ker} \varphi$. We conclude that $J$ and $\operatorname{ker} \varphi$ agree in degrees $\geq n_{0}$.

(3) Let $\varphi: A \rightarrow B=B(X, \mathcal{L}, \sigma)$ be the morphism constructed in part (1) above, and set $A^{\prime}=\varphi(A)$. Let $\mathcal{K}$ be the sheaf of total quotient rings of $X$, and let $R=\mathrm{H}^{0}(\mathcal{K})$ be the function ring of $X$, with induced automorphism also called $\sigma$. Then we may write $B=\bigoplus_{n=0}^{\infty} \mathrm{H}^{0}\left(X, \mathcal{L}_{n}\right) t^{n} \subset Q=R\left[t^{ \pm 1} ; \sigma\right]$. We may also write $A^{\prime}=\bigoplus_{n=0}^{\infty} V_{n} t^{n}$, for some $V_{n} \subset \mathrm{H}^{0}\left(X, \mathcal{L}_{n}\right)$.

As we saw in part (1), $\mathcal{L}_{n_{0}} \cong \mathcal{M}_{n_{0}}$. The sheaf $\mathcal{M}_{n_{0}}$ is very ample on $X=X_{n_{0}}$ since it is the restriction to $X$ of the very ample sheaf $\mathcal{O}(1,1, \ldots, 1)$ on $\mathbb{P}^{\times n_{0}}$. For $n \geq n_{0}$, by the construction of $\varphi$ we have that $V_{n}$ is exactly the image of the map

$$
\mathrm{H}^{0}\left(\mathbb{P}^{\times n}, \mathcal{O}(1,1, \ldots, 1)\right) \stackrel{i_{n}^{*}}{\rightarrow} \mathrm{H}^{0}\left(X_{n}, \mathcal{M}_{n}\right) \stackrel{\left(\phi_{n, n_{0}}\right)_{*}}{\rightarrow} \mathrm{H}^{0}\left(X, \mathcal{L}_{n}\right) .
$$

Since $\phi_{n, n_{0}}$ is an isomorphism for $n \geq n_{0}$, it follows that for $n \geq n_{0}$ the sections in $V_{n}$ generate the sheaf $\mathcal{L}_{n}$ on $X$, and moreover that the corresponding map $X \rightarrow \mathbb{P}\left(V_{n}\right)$ is a closed embedding. By Lemma 3.4 $A^{\prime}$ is a big subalgebra of $Q$.

Now suppose that $\sigma$ is not quasi-unipotent. Then by Proposition 3.5 we have that every big finitely graded subalgebra of $Q$ has exponential growth. In particular, then $A^{\prime}$ has exponential growth, so necessarily $A$ has exponential growth, contradicting the hypothesis.

Thus $\sigma$ must be quasi-unipotent, and since $\mathcal{L}_{n_{0}}$ is very ample on $X, \mathcal{L}$ must be $\sigma$-ample [Ke1, Theorem 1.3]. Now we have all of the needed hypotheses to apply Lemma 4.3 to the injection $A^{\prime} \rightarrow B$. We conclude that $\left(A^{\prime}\right)_{n}=B_{n}$ for $n \gg 0$, in other words $\varphi$ is a surjection in large degree as required.

(4) Given the ring $A$, we constructed the "point scheme data" $X_{n}, i_{n}, \phi_{n}, \psi_{n}, \mathcal{M}_{n}$ using a fixed presentation $A \cong T / I$, where $T=k\left\langle x_{0}, \ldots, x_{d}\right\rangle$ and $\operatorname{dim} A_{1}=d+1$. Given a different presentation $A \cong T / I^{\prime}$, there is some linear transformation $T_{1} \rightarrow$ $T_{1}$ which induces an automorphism $\delta: T \rightarrow T$ such that $I^{\prime}=\delta(I)$. From this it quickly follows that up to isomorphism the point scheme data does not depend on the choice of presentation.

Thus the map $\varphi$ we constructed above depends only on the given ring $A$ and possibly the choice of integer $n_{0}$. If we choose some different $n_{1}$ instead, such that $\phi_{n}$ and $\psi_{n}$ are isomorphisms for all $n \geq n_{1}$, then the construction produces alternative data $X^{\prime}, \mathcal{L}^{\prime}, \sigma^{\prime}$ and a map $\varphi^{\prime}: A \rightarrow B\left(X^{\prime}, \mathcal{L}^{\prime}, \sigma^{\prime}\right)$. If $n_{1}>n_{0}$ then set $\tau=\phi_{n_{1}, n_{0}}$, while if $n_{1}<n_{0}$ then set $\tau=\left(\phi_{n_{0}, n_{1}}\right)^{-1}$. In either case $\tau: X^{\prime} \rightarrow X$ is an isomorphism, $\mathcal{L}^{\prime}=\tau^{*}(\mathcal{L})$, and $\sigma^{\prime}=\tau^{-1} \sigma \tau$. Thus pullback of sections via $\tau$ defines an isomorphism $\gamma: B(X, \mathcal{L}, \sigma) \rightarrow B\left(X^{\prime}, \mathcal{L}^{\prime}, \sigma^{\prime}\right)$ such that $\gamma \varphi=\varphi^{\prime}$. Thus the $\operatorname{map} \varphi$ is canonically determined.

We can now derive Theorem 1.1 as a special case of the result just proved.

Proof of Theorem 1.1. Assume the notation from the beginning of this section, and let $A$ be a strongly noetherian graded algebra generated in degree 1 . The Hilbert scheme theorem [AZ2, Corollary E4.5] shows that since $A$ is strongly noetherian, the morphisms of schemes $\phi_{n}: X_{n+1} \rightarrow X_{n}$ are isomorphisms for all $n \gg 0$. As 
is shown in [KRS, Proposition 10.2], by considering left instead of right modules, the strong noetherian property for $A$ also implies that the maps $\psi_{n}: X_{n+1} \rightarrow X_{n}$ are isomorphisms for all $n \gg 0$. Then we may choose $n_{0}$ such that $\phi_{n}$ and $\psi_{n}$ are isomorphisms for all $n \geq n_{0}$, and then $X=X_{n_{0}}$ is the point scheme for $A$. Since $A$ is noetherian, [SZ] Theorem 0.1] shows that $A$ has subexponential growth. Now all of the hypotheses of Theorem 4.4 are satisfied, and so there exists a ring homomorphism $\varphi: A \rightarrow B(X, \mathcal{L}, \sigma)$. By Theorem 4.4] 4), this homomorphism is canoncially determined.

We now prove several consequences of the main theorem. See [AZ1] for the definition of a noncommutative projective scheme and the definitions of "cohomological dimension" and " $\chi$-condition".

Corollary 4.6. Let $A$ be a strongly noetherian connected graded algebra generated in degree 1. Let $\varphi: A \rightarrow B(X, \mathcal{L}, \sigma)$ be the map given by Theorem [1.1.

(1) Suppose that $A \subset Q=R\left[t^{ \pm 1} ; \tau\right]$, where $R$ is a commutative algebra, and that there is a unit $u \in R$ with $u t \in A_{1}$. Then the map $\varphi$ is an isomorphism in large degree. In particular, there is an isomorphism of noncommutative projective schemes $\operatorname{proj} A \cong\left(\operatorname{coh} X, \mathcal{O}_{X}\right)$.

(2) Suppose that $A$ has a faithful $k$-point module $M$. If $A^{(n)}$ is prime for all $n \geq 2$, Then $A$ is a domain.

(3) Let $M$ be a point module for $A$, and let $A^{\prime}=A / \mathrm{r} \cdot \operatorname{ann}(M)$. Then $A^{\prime}$ has finite GK-dimension, finite Krull dimension, finite cohomological dimension, and satisfies the $\chi$-condition.

Proof. (1) Since $R$ is commutative, we may define an $A \otimes R$-module structure on $Q$ by the rule

$$
q \cdot(a \otimes r)=r q a
$$

for all $q \in Q, a \in A$ and $r \in R$.

For all $n \geq 0$ we have $Q_{n}\left(A_{1}\right) \supset R t^{n} u t=R u^{\sigma^{n}} t^{n+1}=R t^{n+1}=Q_{n+1}$. Thus $M:=Q>0$ is a cyclic $A \otimes R$-module and so is an $R$-point module for $A$. Since $A$ embeds in $M$, we have r. $\operatorname{ann}_{A} M=0$. By Theorem 1.11 1$),(\operatorname{ker} \varphi)_{n}=0$ for $n \gg 0$; by Theorem 1.1(2) $\varphi$ is also surjective in large degree. Then $A$ and $B$ are isomorphic in large degree and $\operatorname{proj} A \cong \operatorname{proj} B$. Further, it is known that $\operatorname{proj} B \cong\left(\operatorname{coh} X, \mathcal{O}_{X}\right)$ AV Theorem 3.12].

(2) If $M$ is a faithful point module for $A$, then it is critical of GK-dimension 1. Hence $A$ is graded prime, hence prime as an ungraded ring. Since $A$ has a faithful point module, the map $\varphi: A \rightarrow B:=B(X, \mathcal{L}, \sigma)$ is bijective in large degree by Theorem 1.1. Since $A$ is prime, $\varphi$ is injective in all degrees. It remains to show that $B$ is a domain. For this it suffices to show that $X$ is integral.

Since the Veronese ring $A^{(n)}$ is prime, so is $B^{(n)}$ for all $n \geq 2$. Note that $B^{(n)} \cong B\left(X, \mathcal{L}_{n}, \sigma^{n}\right)$. Without changing $X$, we may replace $B$ by $B^{(n)}$ and $\sigma$ by $\sigma^{n}$. By doing so we may assume that $\sigma$ fixes all of the irreducible components of $X$. Since $B$ is prime, $X$ has only one irreducible component by [AS Theorem 4.4]. It follows by another application of [AS, Theorem 4.4] that $X$ is reduced, so it is integral.

(3) Replacing $A$ by $A^{\prime}$ we may assume that $A$ has a faithful point module. Then by Theorem 1.1 the map $\varphi: A \rightarrow B:=B(X, \mathcal{L}, \sigma)$ is bijective in large degree. It is known that $B$ has finite GK-dimension, finite Krull dimension, finite cohomological dimension, and satisfies the $\chi$-condition (see [Ke1, Theorem 1.2], 
[AV, Thoerem 3.12], and AZ1 Theorem 4.5, Theorem 7.4]). Then $A$ also has all of these properties.

Proof of Theorem 1.2. Let $A$ be semiprime, strongly noetherian, connected graded and generated in degree 1 , with graded ring of fractions of the form $Q=R\left[t^{ \pm 1} ; \tau\right]$ where $R$ is commutative. Then by the graded Goldie's theorem $A$ has a homogeneous nonzerodivisor of some degree $n \geq 1$, so $Q A_{n}=Q$. Since $Q$ is graded artinian and $A_{n}=\left(A_{1}\right)^{n}$, it follows that $Q A_{1}=Q$. This shows that $Q_{\geq 0}$ is generated by $Q_{0}$ as an $A \otimes R$-module and hence is an $R$-point module for $A$. Now just as in the proof of Corollary 4.6 (1), this implies that the canonical map $\varphi$ is an isomorphism in large degree.

Remark 4.7. It would be interesting to know what happens when the hypotheses of Theorem 1.1 and Theorem 1.2 are weakened. Suppose we start with a ring $A$ which is generated in degree 1 but is only noetherian rather than strongly noetherian. In this case a projective point scheme $X$ may not exist [Ro, [KRS, and there is no hope of producing a canonical homomorphism to a twisted ring by the same method. It is possible there may be an analog of some parts (e.g., part (2)) of Corollary 4.6 for noetherian but not strongly noetherian rings, but any classification result along the lines of Theorem 1.2 will necessarily include more exotic examples such as the nä̈ve blowups defined in $\mathrm{KRS}$. One might also try to relax the hypothesis of generation in degree 1 in Theorem 1.1 while keeping the strong noetherian hypothesis. In this case the point scheme $X$ exists but it is not clear how to define the automorphism $\sigma$ in general. Still, in the special case of graded rings of GK-dimension 2 which are strongly noetherian but not generated in degree 1 , it is known that (possibly after replacing by a Veronese subring) such rings are idealizer rings inside twisted rings $[\mathrm{AS}$. So there is hope that some homomorphism to a twisted ring may still be defined in general regardless of generation in degree 1.

\section{Rings $Q$ OF Finite GK-TyPE}

Let $R$ be a commutative algebra with automorphism $\sigma$ and let $Q$ be the skew Laurent polynomial ring $R\left[t^{ \pm 1} ; \sigma\right]$. Suppose $Q$ is of finite GK-type (see Definition 2.11). Question 1.5 asks:

(1) Does every big finitely graded subring of $Q$ have the same GK-dimension?

(2) Must the algebra $Q$ itself has finite GK-dimension?

Our aim in this last section is to give positive answers to both of these questions in a special case, which was stated in Theorem 1.6 Throughout this section we will restrict our attention to the following situation.

Hypothesis 5.1. Let $k$ be an algebraically closed field, and let $X$ be an integral projective $k$-scheme with quasi-unipotent automorphism $\sigma$. Let $\mathcal{K}$ be the sheaf of total quotient rings of $X$, which is the constant sheaf on the function field $K=$ $k(X)$. Let $Q=K\left[t^{ \pm 1} ; \sigma\right]$, where the automorphism $\sigma$ of $K$ is induced by the automorphism $\sigma$ of $X$.

We claim that under Hypothesis 5.1 the ring $Q$ is of finite GK-type. Indeed, if $\mathcal{L}$ is a very ample invertible sheaf on $X$, then $\mathcal{L}$ is $\sigma$-ample [Ke1, Theorem 1.3] and the ring $B=B(X, \mathcal{L}, \sigma)$ has finite GK-dimension [Ke1, Theorem 6.1]. Since the $\operatorname{ring} B$ is big in $Q$ by Lemma 3.4 $Q$ is of finite GK-type by Proposition 1.4 
We note that given an automorphism $\sigma$ of a field $K$ of finite transcendence degree over $k$, it is possible that there does not exist any projective model $X$ of $K$ with a regular automorphism $\tau \in \operatorname{Aut}(X)$ inducing the map $\sigma$ on rational functions. In this case, we call $\sigma$ non-geometric. We have verified Question 1.5(2) for some specific examples in the non-geometric case, but the details are omitted here. In general Question [1.5] is open for non-geometric $\sigma$.

We start with some easy lemmas and assume Hypothesis 5.1 throughout.

Lemma 5.2. Let $V$ be a finite-dimensional $k$-subspace of $K$. Then there is some very ample invertible sheaf $\mathcal{L} \subset \mathcal{K}$ such that $V \subset \mathrm{H}^{0}(\mathcal{L}) \subset K$.

Proof. Let $\mathcal{M} \subset \mathcal{K}$ be any very ample invertible sheaf on $X$, and let $W=\mathrm{H}^{0}(\mathcal{M})$. By Lemma 3.4 we can choose $u \in W$ such that $K$ is the field of fractions of $k[U]$, where $1 \in U=u^{-1} W$. Putting the elements of $V$ over a common denominator $z$, we have $V \subset z^{-1} U^{n}$ for some $n \geq 0$. Then $\mathcal{L}=z^{-1} u^{-n} \mathrm{H}^{0}\left(\mathcal{M}^{\otimes n}\right) \mathcal{O}_{X}$ is a very ample sheaf (isomorphic to $\mathcal{M}^{\otimes n}$ ) such that $V \subset z^{-1} u^{-n} W^{n} \subset \mathrm{H}^{0}(\mathcal{L})$.

Lemma 5.3. Let $A=\bigoplus_{n=0}^{\infty} V_{n} t^{n}$ be a big finitely graded subalgebra of $Q$. Let $W$ be a finite-dimensional $k$-subspace of $K$. Then there is $0 \neq u \in K$ and $n \geq 1$ such that $W u \subseteq V_{n}$.

Proof. Since $Q$ is of finite GK-type, $A$ has finite GK-dimension. Since $A$ is a domain, the set of all nonzero homogeneous elements of $A$ is an Ore set KL, Proposition 4.13]. Since $A$ is big in $Q$, the graded ring of fractions of $A$ must be $K\left[\left(t^{m}\right)^{ \pm 1} ; \sigma^{m}\right]$ for some $m$ by Lemma [2.3 2 . Now using the Ore condition for $A$, we may write the elements in $W$ over a common denominator. So for some $n \geq 1$ and $z \in A_{n}, W \subseteq A_{n} z^{-1}$. This means that $W u \subseteq V_{n}$ for $u=z t^{-n} \in K$.

Lemma 5.4. Let $\mathcal{L}$ be a very ample invertible sheaf on $X$. If $A$ is the subring of $B=B(X, \mathcal{L}, \sigma)$ generated by $B_{1}$, then $A_{n}=B_{n}$ for all $n \gg 0$.

Proof. Fix some embedding $\mathcal{L} \subset \mathcal{K}$ and write $B=\bigoplus_{n=0}^{\infty} \mathrm{H}^{0}\left(X, \mathcal{L}_{n}\right) t^{n} \subset Q$. Let $A=\bigoplus_{n=0}^{\infty} V_{n} t^{n}$. Since $\mathcal{L}$ is very ample, it is generated by its global sections $V_{1}$, and determines an embedding $X \rightarrow \mathbb{P}\left(V_{1}\right)$. Then for each $n \geq 1, \mathcal{L}_{n}$ is generated by $V_{n}=V_{1} V_{1}^{\sigma} \cdots V_{1}^{\sigma^{n-1}}$, and the sections $V_{n}$ determine an embedding $X \rightarrow \mathbb{P}\left(V_{n}\right)$. Since $\sigma$ is quasi-unipotent, $\mathcal{L}$ is $\sigma$-ample [Ke1, Theorem 1.3], so all of the hypotheses of Lemma 4.3 are verified and that result gives $A_{n}=B_{n}$ for $n \gg 0$.

Now we see how to compare an arbitrary big subalgebra of $Q$ with twisted rings inside $Q$.

Proposition 5.5. Let $A$ be a big connected finitely graded subring of $Q$.

(1) There is a very ample sheaf $\mathcal{L} \subset \mathcal{K}$ such that $A \subset B(X, \mathcal{L}, \sigma) \subset Q$.

(2) There is a very ample sheaf $\mathcal{M}$ and $h \geq 1$ such that $B\left(X, \mathcal{M}, \sigma^{h}\right) \subset A^{(h)}$.

Proof. (1) Write $A=\bigoplus_{n=0}^{\infty} V_{n} t^{n}$. If $A$ is generated by elements of degree less than $n$, Then $A$ is contained in the connected graded ring generated in degree 1 by $V t$, where $V=k+\sum_{i=1}^{n} V_{i}$. Now by Lemma [5.2 we can find a very ample invertible sheaf $\mathcal{L} \subset \mathcal{K}$ such that $V \subseteq \mathrm{H}^{0}(\mathcal{L})=W$. Then

$$
A \subseteq k[V t] \subseteq k[W t] \subseteq B(X, \mathcal{L}, \sigma)=\bigoplus_{n=0}^{\infty} \mathrm{H}^{0}\left(\mathcal{L}_{n}\right) t^{n} \subseteq Q .
$$


(2) Pick an arbitrary very ample invertible sheaf $\mathcal{L}^{\prime} \subset \mathcal{K}$, and let $W^{\prime}=\mathrm{H}^{0}\left(\mathcal{L}^{\prime}\right) \subset$ $K$. By Lemma 5.3, we have $W^{\prime} u \subseteq V_{n}$ for some $n \geq 1$ and some $0 \neq u \in K$. Write $W=W^{\prime} u$. Then let $\mathcal{L}=W \mathcal{O}_{X} \subset \mathcal{K} ;$ so $\mathcal{L}$ is a very ample sheaf isomorphic to $\mathcal{L}^{\prime}$ but embedded differently in $\mathcal{K}$.

Let $C$ be the subring of $B=B\left(X, \mathcal{L}, \sigma^{n}\right) \subset K\left[s ; \sigma^{n}\right]$ generated by $B_{1}$. Then

$$
C_{m}=W W^{\sigma^{n}} \ldots W^{\sigma^{(m-1) n}} s^{m} \subset V_{n} V_{n}^{\sigma^{n}} \ldots V_{n}^{\sigma^{(m-1) n}} s^{m} \subset V_{n m} s^{m}
$$

for all $m$ and so we have an inclusion of connected graded rings $C \subset A^{(n)}=$ $\bigoplus_{m \geq 0} A_{m n}$. Since $\sigma$ is quasi-unipotent by hypothesis, the automorphism $\sigma^{n}$ is also quasi-unipotent. Then by Lemma [5.4 $C$ and $B$ are equal in large degree, say in degrees $\geq p$. Therefore passing to a further Veronese subring we have an inclusion $B^{(p)}=B\left(X, \mathcal{M}, \sigma^{p n}\right) \subset A^{(n p)}$, where $\mathcal{M}=\mathcal{L} \otimes \mathcal{L}^{\sigma^{n}} \otimes \cdots \otimes \mathcal{L}^{\sigma^{n(p-1)}}$ is very ample.

The following is a result of Keeler [Ke1].

Lemma 5.6. The number $\operatorname{GKdim} B\left(X, \mathcal{N}, \sigma^{n}\right)$ is the same positive integer for every ample invertible sheaf $\mathcal{N}$ and all $n \geq 1$.

Proof. Since by Hypothesis [5.1 $\sigma$ is quasi-unipotent, this follows from [Ke1, $\S 6]$ : Theorem 6.1, Equation 6.3, Lemma 6.5(2) and Lemma 6.8.

We are now ready to prove the main result of this section.

Proof of Theorem [1.6. Note that the hypotheses of the theorem allow us to assume Hypothesis 5.1 Let $d=\operatorname{GKdim} B(X, \mathcal{L}, \sigma)$.

(1) Let $A$ be any big finitely graded subalgebra of $Q$. Since the ring $k+A_{\geq 1}$ has the same GK-dimension, we may assume that $A$ is connected. Since $A^{(h)} \subset A$ as ungraded rings, by Proposition 5.5 for some $h$ and very ample sheaves $\mathcal{N}, \mathcal{M}$ we have

$\operatorname{GKdim} B\left(X, \mathcal{M}, \sigma^{h}\right) \leq \operatorname{GKdim} A^{(h)} \leq \operatorname{GKdim} A \leq \operatorname{GKdim} B(X, \mathcal{N}, \sigma)$,

where the two ends are equal to $d$ by Lemma 5.6. Thus GKdim $A=d$.

(2) Clearly $\operatorname{GKdim} Q \geq \operatorname{GKdim} A=d$. It remains to $\operatorname{show}$ that $\operatorname{GKdim} Q \leq$ $d+\operatorname{dim} X$.

Let $V$ be a finite-dimensional $k$-subspace of $Q$. There is a finite-dimensional $W \subseteq$ $K$ and $m \geq 0$ such that $V \subseteq V^{\prime}:=\sum_{i=-m}^{m} W t^{i}$; we may choose $W$ so that $1 \in W$, and so that $W=\mathrm{H}^{0}(\mathcal{L})$ for some very ample invertible sheaf $\mathcal{L} \subset \mathcal{K}$, by Lemma 5.2 We want to give an upper bound for GKdim $k\left[V^{\prime}\right]$. Setting $V^{\prime \prime}=W t^{-1}+W+W t$, then $\left(V^{\prime \prime}\right)^{n} \supset V^{\prime}$, so GKdim $k\left[V^{\prime}\right] \leq \mathrm{GKdim} k\left[V^{\prime \prime}\right]$ and it is enough to bound the latter dimension.

By the fact that $1 \in W$ we have

$$
\left(V^{\prime \prime}\right)^{n} \subseteq \sum_{i=-n}^{n}\left(W^{\sigma^{-n}} W^{\sigma^{-n+1}} \ldots W^{\sigma^{n}}\right)^{n} t^{i} .
$$

Note also that

$$
\left(W^{\sigma^{-n}} W^{\sigma^{-n+1}} \cdots W^{\sigma^{n}}\right)^{n} \subseteq \mathrm{H}^{0}\left(\left(\mathcal{L}^{\sigma^{-n}} \otimes \mathcal{L}^{\sigma^{-n+1}} \otimes \cdots \otimes \mathcal{L}^{\sigma^{n}}\right){ }^{\otimes n}\right) \cong \mathrm{H}^{0}\left(\mathcal{L}_{2 n+1}^{\otimes n}\right)
$$

So altogether we may conclude that

$$
\operatorname{dim}_{k}\left(V^{\prime \prime}\right)^{n} \leq(2 n+1) \operatorname{dim}_{k} \mathrm{H}^{0}\left(\mathcal{L}_{2 n+1}^{\otimes n}\right)
$$


Now for each $n \geq 0$ write $e_{n}=\operatorname{dim}_{k} \mathrm{H}^{0}\left(\mathcal{L}_{n}\right), f_{n}=(2 n+1) \operatorname{dim}_{k} \mathrm{H}^{0}\left(\mathcal{L}_{2 n+1}^{\otimes n}\right)$, and $g_{n}=\operatorname{dim}_{k} \mathrm{H}^{0}\left(\mathcal{L}_{n}^{\otimes n}\right)$. Since $B=B(X, \mathcal{L}, \sigma)$ is a finitely generated domain, we have $d=\operatorname{GKdim} B=\left(\overline{\lim } \log _{n} e_{n}\right)+1$. It follows from (5.7) that GKdim $k\left[V^{\prime \prime}\right] \leq$ $\varlimsup \log _{n} f_{n}$. Since $\mathcal{L}$ is very ample, we have $\operatorname{dim}_{k} \mathrm{H}^{0}\left(\mathcal{L}_{2 n+1}^{\otimes n}\right)<\operatorname{dim}_{k} \mathrm{H}^{0}\left(\mathcal{L}_{2 n+1}^{\otimes 2 n+1}\right)$, and so $\varlimsup_{\lim } \log _{n} f_{n}=\left(\mp \log _{n} g_{n}\right)+1$. So we will be done if we can show that $g_{n} \leq n^{\operatorname{dim} X} e_{n}$ for $n \gg 0$.

Since $\mathcal{L}$ is very ample, for $n \gg 0$ we have $\mathrm{H}^{q}\left(\mathcal{L}^{\otimes n} \otimes \mathcal{M}\right)=0$ for any ample sheaf $\mathcal{M}$ and all $q>0[\mathrm{Fj}$, p. 520, Theorem 1]. This implies that, for $n \gg 0$, $\mathrm{H}^{q}\left(\mathcal{L}_{n}^{\otimes n}\right)=0$. Also, $\mathrm{H}^{q}\left(\mathcal{L}_{n}\right)=0$ for $n \gg 0$ and all $q>0$ since $\mathcal{L}$ is $\sigma$-ample by [Ke1, Theorem 1.3]. For a coherent sheaf $\mathcal{F}$ on $X$, define $\chi(\mathcal{F})=\bigoplus_{q=0}^{\infty}(-1)^{q} \operatorname{dim}_{k} \mathrm{H}^{q}(\mathcal{F})$. So $g_{n}=\chi\left(\mathcal{L}_{n}^{\otimes n}\right)$ and $e_{n}=\chi\left(\mathcal{L}_{n}\right)$ for $n \gg 0$.

Now, as in Ke1, p. 529], the Riemann-Roch formula may be used to show for an invertible sheaf $\mathcal{M}$ that

$$
\chi(\mathcal{M})=\sum_{j=0}^{\operatorname{dim} X} \sum_{V \in A_{j}} a_{V} \overbrace{(\mathcal{M} . \mathcal{M} . \cdots . \mathcal{M})_{V}}^{j},
$$

where $A_{j}$ is some finite set of $j$-dimensional subvarieties of $X$ and the $a_{V}$ are some rational numbers. All that we need to know about the intersection form appearing in this formula is that it is multilinear (with respect to addition of divisors, which corresponds to tensor product of sheaves). So we conclude for all $n \geq 0$ that

$$
\chi\left(\mathcal{L}_{n}^{\otimes n}\right)=\sum_{j=0}^{\operatorname{dim} X} n^{j} \sum_{V \in A_{j}} a_{V}\left(\left(\mathcal{L}_{n}\right)^{j}\right)_{V} \leq n^{\operatorname{dim} X} \chi\left(\mathcal{L}_{n}\right)
$$

and so $g_{n} \leq n^{\operatorname{dim} X} e_{n}$ for $n \gg 0$ as we wished.

The lower bound in Theorem 1.6 (2) holds when $\sigma=I d$ (or $\sigma$ has finite order). We can show that the upper bound is achieved in some specific examples, for instance if $X=\mathbb{P}^{n}$ and $\sigma$ is a generic automorphism. In general it is not clear exactly how the value of $\operatorname{GKdim} Q$ depends on the properties of the automorphism $\sigma$.

To close, we show that as a corollary of Theorem 1.6 we can obtain the exact value of the Gelfand-Kirillov transcendence degree of the division rings of fractions of twisted rings. The GK-transcendence degree of an algebra $A$ is defined to be

$$
\operatorname{GKtr} A=\sup _{V}\left(\inf _{z} \operatorname{GKdim} k[z V]\right)
$$

where $V$ ranges over all finite-dimensional subspaces of $A$ containing 1 and $z$ ranges over all nonzerodivisors of $A$. This is a very useful invariant for division rings, but it is difficult to calculate in general GK, Zh.

Corollary 5.8. Let $B=B(X, \mathcal{L}, \sigma)$ where $\mathcal{L}$ is $\sigma$-ample, and let $D$ be the division ring of fractions of the noetherian domain $B$. If $A$ is any algebra with $B \subset A \subset D$, then GKtr $A=\operatorname{GKdim} B$.

Proof. (1) By [Zh Propositions 2.1 and 3.1(3)],

$$
\text { GKtr } D \leq \operatorname{GKtr} A \leq \mathrm{GKtr} B \leq \mathrm{GKdim} B .
$$

It remains to show that $\operatorname{GKtr} D \geq \operatorname{GKdim} B$. Let $\nu: B \rightarrow B$ be the valuation defined by the grading of $B$. By [Zh, Proposition 6.5], $\nu$ can be extended to a valuation $\nu: D \rightarrow Q$ where $Q=K\left[t^{ \pm 1} ; \sigma\right]$ is the graded ring of fractions of $B$. Let $V$ be a finite-dimensional subspace of $K$ containing 1 such that $K$ is the 
field of fractions of $k[V]$. Set $W=V t+V+V t^{-1}$. It will suffice to show that GKdim $k[z W] \geq \operatorname{GKdim} B$ for all nonzero $z \in Q$. By [Zh Theorem 6.7], we only need to show that $\operatorname{GKdim} k[\nu(z W)]=\operatorname{GKdim} k[\nu(z) W] \geq \operatorname{GKdim} B$. Thus we can assume that $z$ is a homogeneous element of $Q$, and we write $z=q t^{n}$ for some $q \in K$ and $n \in \mathbb{Z}$. If $n \geq 0$, then $k[z W]$ contains the connected $\mathbb{N}$-graded algebra $C=k\left[q V t^{n+1}\right]$, which is big in $Q$ by definition. Then $\operatorname{GKdim} C=\operatorname{GKdim} B$ by Theorem [1.6] Otherwise $n<0$, and $k[z W]$ contains the algebra $D=k\left[q V t^{n}\right]$. The ring $D$ has the same GK-dimension as the big $\mathbb{N}$-graded algebra $C=k\left[q V t^{-n}\right]$, and again $\operatorname{GKdim} C=\operatorname{GKdim} B$. In either case we have that $\operatorname{GKdim} k[z W] \geq$ $\operatorname{GKdim} B$.

\section{ACKNOWLEDGMENTS}

We thank Mike Artin, Dennis Keeler, Zinovy Reichstein, Paul Smith, and Toby Stafford for helpful conversations.

\section{REFERENCES}

[Ar] D. Arapura, Frobenius amplitude and strong vanishing theorems for vector bundles, Duke Math. J. 121 (2004), no. 2, 231-267, with an appendix by Dennis S. Keeler.

[AS] M. Artin and J.T. Stafford, Noncommutative graded domains with quadratic growth, Invent. Math. 122 (1995), no. 2, 231-276.

[ASZ] M. Artin, L.W. Small and J.J. Zhang, Generic flatness for strongly Noetherian algebras, J. Algebra 221 (1999), no. 2, 579-610.

[ATV] M. Artin, J. Tate and M. Van den Bergh, Some algebras associated to automorphisms of elliptic curves, The Grothendieck Festschrift, Vol. I, 33-85, Progr. Math., 86, Birkhäuser Boston, Boston, MA, 1990.

[AV] M. Artin and M. Van den Bergh, Twisted homogeneous coordinate rings, J. Algebra 133 (1990), no. 2, 249-271.

[AZ1] M. Artin and J.J. Zhang, Noncommutative projective schemes, Adv. Math. 109 (1994), no. $2,228-287$.

[AZ2] M. Artin and J.J. Zhang, Abstract Hilbert schemes, Algebr. Represent. Theory 4 (2001), no. 4, 305-394.

[Fj] T. Fujita, Vanishing theorems for semipositive line bundles, Algebraic geometry (Proceedings, Tokyo/Kyoto, 1982), Lecture Notes in Math., vol. 1016, Springer, Berlin, 1983, pp. 519-528.

[GK] I.M. Gelfand and A.A. Kirillov, Sur les corps liés aux algèbres enveloppantes des algèbres de Lie. (French) Inst. Hautes Études Sci. Publ. Math. No. 31, 1966 5-19.

[Ha] R. Hartshorne, Algebraic geometry, Springer-Verlag, New York, 1977, Graduate Texts in Mathematics, No. 52.

[Ke1] D. S. Keeler, Criteria for $\sigma$-ampleness, J. Amer. Math. Soc. 13 (2000), no. 3, 517-532

$[\mathrm{Ke} 2]$ D.S. Keeler, Ample filters and Frobenius amplitude, in preparation.

[KL] G. R. Krause and T. H. Lenagan, Growth of algebras and Gelfand-Kirillov dimension, revised ed., American Mathematical Society, Providence, RI, 2000.

[KRS] D.S. Keeler, D. Rogalski and J.T. Stafford, Naive noncommutative blowing up, to appear in Duke Math J.

[Na] Y. Nakai, Some fundamental lemmas on projective schemes, Trans. Amer. Math. Soc. 109 (1963), 296-302.

[Ro] D. Rogalski, Generic noncommutative surfaces, Adv. Math. 184 (2004), no. 2, 289-341.

[RRZ] Z. Reichstein, D. Rogalski, and J.J. Zhang, Projectively Simple Rings, preprint, 2004, available at www.arxiv.org/math.RA/0401098.

[SV] B. Shelton and M. Vancliff, Embedding a quantum rank three quadric in a quantum $\mathbf{P}^{3}$, Comm. Algebra 27 (1999), no. 6, 2877-2904.

[SZ] D.R. Stephenson and J. J. Zhang, Growth of graded Noetherian rings, Proc. Amer. Math. Soc. 125 (1997), no. 6, 1593-1605.

[Va] M. Vancliff, Quadratic algebras associated with the union of a quadric and a line in $\mathbf{P}^{3}$, J. Algebra 165 (1994), no. 1, 63-90. 
[VV1] M. Vancliff and K. Van Rompay, Embedding a quantum nonsingular quadric in a quantum $\mathbf{P}^{3}$, J. Algebra 195 (1997), no. 1, 93-129.

[VV2] _ Four-dimensional regular algebras with point scheme, a nonsingular quadric in $\mathbf{P}^{3}$, Comm. Algebra 28 (2000), no. 5, 2211-2242.

[Zh] J.J. Zhang, On Gelfand-Kirillov transcendence degree, Trans. Amer. Math. Soc. 348 (1996), no. $7,2867-2899$.

(Rogalski) Department of Mathematics, Mit, Cambridge, MA 02139-4307, USA

E-mail address: rogalski@math.mit.edu

(Zhang) Department of Mathematics, Box 354350, University of Washington, SeatTLE, WA 98195, USA

E-mail address: zhang@math.washington.edu 